\title{
Presencia equilibrada entre mujeres y hombres en consejos de administración: identificación de los Stakeholders
}

\section{Gender balance on boards: Stakeholders identification}

\author{
Pilar de Luis CARnicer ${ }^{1}$ \\ Estrella Bernal Cuenca ${ }^{2}$ \\ María José Vela Jiménez ${ }^{2}$ \\ Manuela Pérez PÉrez ${ }^{1}$ \\ Universidad de Zaragoza (España)
}

Recibido el 10 de junio de 2010, aceptado el 31 de mayo de 2011

$\mathrm{N}^{\circ}$ de clasificación JEL: M100, M190, M140

DOI: $10.5295 / \mathrm{cdg} .100243 \mathrm{pd}$

\section{Resumen:}

En España, la reciente normativa ha convertido la presencia equilibrada de mujeres y hombres en los Consejos de Administración en un elemento a incorporar en la gestión empresarial. El objetivo de este trabajo es identificar los grupos de interés (stakeholders) que demandan a las empresas esta presencia equilibrada utilizando la metodología de Mitchell et al. (1997) y Agle et al. (1999).

Los resultados nos sugieren que los propietarios son el grupo de mayor importancia y también el primero en cuanto a la valoración de los atributos considerados, lo que nos hace pensar que los objetivos económicos siguen siendo prioritarios en las empresas. Pero también se ha observado que el Gobierno se percibe como muy importante. A pesar de que se le reconoce un gran poder, son la legitimidad y urgencia los atributos que explican la importancia percibida en el Gobierno, lo que nos hace dudar de la efectividad que tendría el desarrollo de una normativa obligatoria, más allá de la recomendación actual.

Palabras clave:

Presencia Equilibrada, Mujeres Directivas, Teoría Stakeholders, Gobierno Corporativo, Responsabilidad Social Corporativa.

\begin{abstract}
:
Recent Spanish law and regulations have turned gender balance on Boards of Directors into a necessary element to manage in companies. The aim of this paper is to identify the stakeholders demanding such gender balance
\end{abstract}

\footnotetext{
${ }^{1}$ Departamento de Economía y Dirección de Empresas Universidad de Zaragoza Centro Politécnico Superior c/ María de Luna, 350018 Zaragoza. pluis@unizar.es, manuela.perez@unizar.es.

${ }^{2}$ Departamento de Economía y Dirección de Empresas Escuela Universitaria de Estudios Empresariales Universidad de Zaragoza Edificio Lorenzo Normante María de Luna, s/n Zaragoza, 50018. bercue@unizar.es, mjvela@unizar.es
} 
Presencia equilibrada entre mujeres y hombres en consejos de administración: identificación de los Stakeholders

to companies, according to the Methodology of Mitchell et al. (1997) and Agle et al. (1999). The results sugest the shareholders are the most important group, so it seems that the financial goals continue being a priority. But we also observe that the Government is perceived as a very important stakeholder. Despite managers acknowledge the Government has a lot of power, its importance is explained by urgency and legitimacy attributes. This fact makes us doubting about the effectiveness of any development of a compulsory law, beyond the current one.

\section{Keywords:}

Gender Balance, Female Managers, Stakeholders Theory, Corporative Government, Corporate Social Responsibility. 


\section{INTRODUCCIÓN}

La presencia equilibrada de mujeres y hombres en los Consejos de Administración de las empresas es actualmente un tema de gran importancia en la agenda política y social (European Comisión 2004, 2005). En España, los avances en igualdad de oportunidades entre hombres y mujeres han sido notables. De acuerdo con The Global Gender Gap Report (Hausmann et al., 2010), España pasó del puesto $25^{\circ}$ en el año 2.000 al puesto $5^{\circ}$ en el 2.010. Sin embargo, la presencia de mujeres en los órganos de poder empresarial es uno de sus puntos débiles. En este apartado, España se posiciona en $43^{\circ}$ lugar. No obstante, el informe predice grandes avances para España debido principalmente a la normativa aprobada en nuestro país en los últimos años.

La Ley 3/2007 de 22 de Marzo para la Igualdad Efectiva de Mujeres y Hombres introdujo cambios que obligan a las empresas e Instituciones Públicas a tomar una posición proactiva ante la igualdad de oportunidades, entre las que cabe destacar la obligación de las empresas de más de 250 trabajadores de elaborar un Plan de Igualdad, o la obligación en las Instituciones Públicas de atender al principio de presencia equilibrada de mujeres y hombres en sus Consejos de Administración. En concreto, la Ley obliga a la Administración Pública a observar el principio de presencia equilibrada ${ }^{1}$ en los nombramientos que le corresponda efectuar en los Consejos de Administración de las empresas en cuyo capital participe (art.54), mientras que alcanzar esta presencia equilibrada es sólo una recomendación para las empresas privadas obligadas a presentar cuenta de pérdidas y ganancias (Art.75) ${ }^{2}$.

El código Conthe de buenas prácticas para empresas cotizadas, también incorporó en el año 2006 la recomendación de realizar un esfuerzo deliberado para incluir, entre los potenciales candidatos, mujeres que reúnan el perfil profesional buscado, obligando al Consejo a explicar los motivos de la escasez o inexistencia del número de consejeras y las iniciativas adoptadas para corregir esta situación.

Todavía parece precipitado hablar sobre los avances que se pueden derivar de estas medidas, sin embargo con los datos disponibles ya podemos vislumbrar algunos cambios (Mateos et al., 2011; Carrasco y Laffarga, 2007; del Brio and del Brio, 2009). En 2006, el porcentaje de consejeras de las empresas del IBEX 35 era del 3,46\%, porcentaje que venía manteniéndose invariable en las décadas anteriores. En 2007, primer año que las empresas cotizadas se vieron obligadas a informar sobre sus acciones, el porcentaje ascendió al 6,43\% y de nuevo en 2008 ascendió al 8,48\% (Instituto de la Mujer, 2011).

En cualquier caso, es necesario señalar que la ley no obliga a las empresas privadas. La ausencia de obligatoriedad nos lleva a pensar en el escaso impacto que puede tener en las decisiones empresariales. Ello implica que la presencia equilibrada queda al arbitrio de las empresas en función de los valores éticos que se imponga la organización y que se explicitan en su declaración de Responsabilidad Social Corporativa.

\footnotetext{
${ }^{1}$ No menos del $40 \%$ ni más del $60 \%$ de cualquiera de los sexos.

${ }^{2}$ Artículo 75. Las sociedades obligadas a presentar cuenta de pérdidas y ganancias no abreviada procurarán incluir en su Consejo de administración un número de mujeres que permita alcanzar una presencia equilibrada de mujeres y hombres en un plazo de ocho años a partir de la entrada en vigor de esta Ley. Lo previsto en el párrafo anterior se tendrá en cuenta para los nombramientos que se realicen a medida que venza el mandato de los consejeros designados antes de la entrada en vigor de esta Ley.
} 
Esta situación parece estar de acuerdo con la tendencia legislativa en Europa, que considera que el ámbito de la Responsabilidad Social Corporativa pertenece a la libre iniciativa empresarial y que no debería ser regulada desde el Estado (soft law) sino por códigos éticos de conducta (Grosser, 2009; Grosser y Moon, 2005, 2008). No obstante, la normativa europea sostiene que la diversidad de género en los Consejos de Administración conlleva efectos positivos en los resultados empresariales y favorece el crecimiento económico, por lo que defiende la presencia equilibrada tanto por criterios económicos como éticos. (Elósegui y Lousada, 2007).

Existe una amplia literatura sobre los efectos positivos que la diversidad de género tiene en los resultados empresariales, sin embargo los resultados son ambiguos. Algunos estudios encuentran relaciones positivas (Adler, 2001; Erhardt, Werbel y Schrader, 2003; Veleva, 2005; Krishnan y Park, 2005), otros negativas (Ryan y Haslam, 2005, 2007) y otros no encuentran ninguna relación (Du Rietz y Hanrekson, 2000; Kochan et al., 2003; Rose, 2007). Si esta relación positiva no puede ser demostrada y la normativa no obliga a las empresas privadas, quedaría una tercera vía para conseguir que las empresas tomen un comportamiento proactivo para alcanzar la presencia equilibrada: que perciban una presión suficiente de los stakeholders para que incluyan estas acciones dentro de sus objetivos estratégicos.

Incluir la "presencia equilibrada" en la Responsabilidad Social Corporativa de las empresas sería un primer paso para convertir la normativa en estrategia corporativa, siempre y cuando las empresas aceptaran utilizar el modelo de gestión que propone la teoría de los stakeholders.

Este artículo presenta un estudio, cuyo objetivo es aportar información sobre cuáles son los stakeholders relevantes a la hora de demandar a las empresas acciones proactivas que lleven a conseguir una presencia equilibrada de mujeres y hombres en los Consejos de Administración.

El apartado segundo presenta el marco teórico en que se fundamentan las hipótesis planteadas y que son contrastadas en el estudio empírico. El apartado tercero presenta el estudio empírico, tanto la metodología utilizada como los principales resultados. Por último se presentan las conclusiones junto con las limitaciones del estudio y propuestas de futuras investigaciones.

\section{MARCO TEÓRICO}

Las investigaciones en torno a la diversidad de género en los Consejos de Administración utilizan dos enfoques: el enfoque financiero y el enfoque ético. El enfoque financiero persigue la eficiencia económica, mientras que desde el enfoque ético el objetivo es conseguir la equidad social.

La mayoría de los estudios utilizan el enfoque financiero para explicar los beneficios que las mujeres podrían aportar al resultado financiero de la empresa. Según este enfoque, el principal objetivo de la empresa es la maximización del beneficio para sus accionistas. Las principales teorías que apoyan este enfoque son dos: la Teoría de Agencia y la Teoría de Recursos. La Teoría de Agencia argumenta que la diversidad puede incrementar la independencia del Consejo porque personas con diferente sexo, así como etnia o cultura, 
pueden plantear cuestiones que provendrían de consejeros con bagajes más tradicionales (Carter et al., 2003). La Teoría de Recursos defiende que las competencias, diferentes y complementarias, que aportan las mujeres son recursos difícilmente imitables para los competidores, lo que las convierte en ventajas competitivas (Schyns y Sanders, 2005). Desde un punto de vista meritocrático la falta de mujeres en los puestos de poder empresarial puede provocar la pérdida de talentos. Si sólo los hombres son candidatos potenciales, la selección de estos puestos se realizará entre una distribución restringida de cualificaciones y, en promedio, esto implicará una calidad muy inferior a la que se conseguirá si los candidatos y candidatas son seleccionados entre los mejores de una distribución compuesta tanto por hombres como por mujeres (Smith et al., 2006).

Diversos autores señalan el reduccionismo del enfoque financiero y plantean la necesidad del enfoque ético en la gestión empresarial, cuyo objetivo sea la maximización del beneficio neto para toda la sociedad, y que contempla la equidad en el reparto de los beneficios entre todos los grupos involucrados en la actividad de la empresa (Cragg, 2002; Greenwood, 2007; Machold et al., 2008; Rodríguez, 2006; Smyth, 2008). La introducción de un enfoque ético en la gestión empresarial se está consolidando gracias a los avances realizados en el campo de la Responsabilidad Social Corporativa (Carroll, 1991) que, apoyada por la teoría de los stakeholders (Freeman, 1984), ha conseguido integrar los objetivos económicos y éticos (Cragg, 2002; Greenwood, 2007; Rodríguez, 2006; Smyth, 2008). El debate académico se centra en estos momentos en la necesidad de definir qué teoría ética debería ser utilizada, habiendo autores que defienden la ética feminista como la más adecuada (Machold et al. 2008; Ely y Padavic, 2007; Syed y Murray, 2008; Burton y Dunn, 1996; Wicks et al., 1994).

Bajo este enfoque, la igualdad de oportunidades entre hombres y mujeres es un aspecto relevante a tener en cuenta en la toma de decisiones empresariales, y conseguir una presencia equilibrada en los Consejos de Administración debería de ser un objetivo a alcanzar ya que puede ser vista como una respuesta a las presiones y demandas de los diferentes stakeholders (Henriques y Sadorsky, 1999). Francoeur et al. (2007) defienden esta idea, incluso si no se consiguiera una mejora de los resultados financieros. Cuando menos puede utilizarse para enviar señales positivas a sus stakeholders (Brammer et al., 2009; Huse y Solverg, 2006). Aunque algunos autores reconocen la utilidad del marco de los stakeholders para el análisis de la diversidad de género en los Consejos de Administración (Dimovski y Brooks, 2008), es escasa la literatura que relaciona directamente ambos conceptos (Grosser y Moon, 2008; Grosser, 2009). Tradicionalmente, los temas relativos al género son considerados demandas del personal de la empresa (Agle et al., 1999).

\subsection{Identificación de los stakeholders ante la presencia equilibrada}

Freeman (1984) definió los stakeholders como "cualquier grupo o individuo que puede afectar o ser afectado por el logro de los propósitos de una corporación. Stakeholders incluye a empleados, clientes, proveedores, accionistas, bancos, ambientalistas, gobierno u otros grupos que puedan ayudar o dañar a la corporación”. Fernández y Nieto (2004) consideran lógico limitar el análisis a un tema específico y determinar el interés de los stakeholders en él, así como la respuesta que cada uno de ellos recibiría desde la dirección. 
Nuestra propuesta trata de identificar a los stakeholders que las empresas consideren importantes con respecto a un tema específico social: la presencia equilibrada de hombres y mujeres en los Consejos de Administración. Esta variable podría considerarse como uno de los indicadores para medir el grado de consecución de los objetivos sociales, puesto que la falta de instrumentos para medir los objetivos no financieros en la empresa, y concretamente la riqueza neta total para todos los partícipes, es considerada una de las deficiencias que tiene la teoría de los stakeholders. (Rodríguez, 2003, p. 111).

Los grupos feministas son frecuentemente nombrados, junto con movimientos de derechos civiles y movimientos medioambientales como stakeholders sociales (Laplume et al., 2008; Carroll, 1991). Sin embargo, mientras que el impacto en la gestión medioambiental ha sido objeto de estudio ampliamente (Reed, 2008), las investigaciones sobre la relación entre stakeholders y gestión de la diversidad son escasas (Grosser y Moon, 2008; Grosser, 2009). Debido a la falta de estudios acerca de los grupos que podrían calificarse de stakeholders en relación con la igualdad de oportunidades entre hombres y mujeres, hemos elegido como referencia inicial los utilizados en relación al medioambiente, y concretamente estudios empíricos realizados con muestras españolas (Díez et al., 2008; Fernández y Nieto, 2004), dado que los stakeholders seleccionados en estos estudios pueden compartir sensibilidades hacia el tema la presencia equilibrada. Esta decisión está basada en el enfoque ecofeminista, desde el que se infiere que los agentes sociales sensibles a aspectos medioambientales de la gestión empresarial, lo serán también a otros aspectos sociales como la igualdad de oportunidades entre mujeres y hombres, ya que el ecofeminismo defiende el paradigma de sostenibilidad que comparten la economía ecológica y la economía feminista (Crittenden, 2000; Pandey, 2010).

En nuestro caso no podemos hablar de las mujeres como un stakeholder, dado que la mujeres suponen alrededor del $50 \%$ de la población en general y por lo tanto van a formar parte de todos los posibles stakeholders considerados: empleadas, consumidoras, directivas, etc. (Grosser, 2009). Ahora bien, dentro de estos grupos de interés la situación de inferioridad de las mujeres se manifiesta con más fuerza. Por ejemplo, su situación de inferioridad como empleadas y principalmente su ausencia en los equipos de gobierno.

Para realizar esta identificación se ha utilizado la aportación de Mitchell et al. (1997), que fue ampliada por Agle et al. (1999). El modelo propone que las demandas de los stakeholders serán atendidas en la medida en que los/as directivos/as los perciban importantes y que esa importancia percibida estará a su vez condicionada por la percepción que tengan sobre tres atributos en cada uno de los grupos de interés considerados: poder para presionar, legitimidad de sus demandas y urgencia en la respuesta que esperan de la empresa. Cada uno de estos tres atributos van a influir de forma individual a aumentar la importancia percibida por las empresas, es decir, cuanto mayor sea el poder que un grupo tenga para presionar, mayor será la importancia percibida de ese grupo por la dirección de la empresa. Igualmente, la importancia aumentará en la medida en que la dirección considere que el grupo tiene derecho a demandar ciertas acciones (legitimidad). O bien, que la dirección de la empresa perciba que ese grupo exige una gran rapidez en la respuesta a sus demandas (urgencia). Cada stakeholder puede tener uno o varios de los tres atributos, de forma que también podremos observar un efecto conjunto de los tres atributos sobre la importancia percibida. Cuanto mayor sea el número de atributos mayor será la importancia de ese grupo. El modelo de Mitchell et al. (1997) ha sido frecuentemente utilizado para analizar 
la importancia de los stakeholders (Laplume et al., 2008). En este estudio intentaremos comprobar que también se cumple cuando los atributos considerados se relacionan con las políticas de igualdad de oportunidades y más concretamente con acciones de búsqueda de presencia equilibrada de mujeres y hombres en los Consejos de Administración. Por lo tanto proponemos, en el tema de presencia equilibrada, las hipótesis planteadas por Agle et al. (1999, p.510):

H1a. El atributo percibido de poder para alcanzar la presencia equilibrada de un stakeholder, influirá positivamente sobre la importancia percibida de ese stakeholder.

$\mathrm{H} 1 \mathrm{~b}$. El atributo percibido de legitimidad para alcanzar la presencia equilibrada, influirá positivamente sobre la importancia percibida de ese stakeholder.

H1c. El atributo percibido de urgencia para alcanzar la presencia equilibrada, influirá positivamente sobre la importancia percibida de ese stakeholder.

H1d. La acumulación en un stakeholder de poder, legitimidad y urgencia para alcanzar la presencia equilibrada estará positivamente asociada con la importancia percibida de ese stakeholder.

\section{ESTUDIO EMPÍRICO}

\subsection{Metodología}

La recogida de datos se realizó a través de un cuestionario dirigido a la dirección de la empresa, que incluía una definición de los atributos mencionados y una relación de diez stakeholders. Se les pidió a los directivos y a las directivas que considerasen y puntuasen estos atributos en una escala de Likert de uno a siete, para cada uno de los diez stakeholders. La definición de los atributos fue la siguiente:

- Poder: Considera que ese stakeholder puede utilizar (tanto si lo hace como si no) cualquier tipo de presión externa, incluso influyendo en su imagen pública, para conseguir que la empresa busque la presencia equilibrada.

- Legitimidad: Considera que las preocupaciones de ese stakeholder sobre la obtención de una presencia equilibrada son adecuadas y/o legítimas.

- Urgencia: Considera que las preocupaciones de ese stakeholder sobre la obtención de una presencia equilibrada conlleva la exigencia de una rápida respuesta a sus demandas.

- Importancia: Considera importante al stakeholder, en función del grado de atención, tiempo y prioridad que demanda sobre la obtención de una presencia equilibrada.

En cuanto a los stakeholders relacionados, se partió de los estudios medioambientales citados (Díez et al., 2008; Fernández y Nieto, 2004), con algunos cambios. Se mantuvieron los cuatro grupos organizacionales usados en estos estudios: Propietarios/as, accionistas; personal de la empresa; clientes y proveedores. Este grupo fue ampliado con el equipo de dirección como un stakeholder relevante. La razón fue la estrecha relación que existe entre la presencia de mujeres en el Consejo de Administración y la presencia de mujeres en la Alta Dirección (Bilimoria, 2006). La presencia de mujeres en los Consejos de Administración puede suponer la existencia de procesos de promoción profesional, desde la alta dirección, justos y no discriminatorios, pero también puede darse el fenómeno inverso, la presencia de mujeres en el Consejo de Administración aumenta las posibilidades de las mujeres de acceder a la Dirección de la empresa (Bilimoria, 2006). 
En cuanto a los grupos organizacionales, se mantuvo el Gobierno como principal stakeholder. Las asociaciones profesionales se sustituyeron por los sindicatos, ya que como instituciones sociales los sindicatos tienen más influencia en los asuntos de promoción de carrera profesional de los empleados de la que tienen las asociaciones profesionales. Se tomó esta decisión porque consideramos que la presencia equilibrada de mujeres en el consejo de administración está muy relacionada con la promoción profesional de las mujeres, hasta el punto en que los temas de igualdad de oportunidades entre hombres y mujeres están incluidos como objetivos a conseguir en beneficio del personal de la empresa en los informes de RCS (Grosser, 2009). Así mismo, en países como Alemania, existe una cogestión sindical de las empresas con representación sindical en los consejos de administración (ley de Comités de Empresa de 1952 y la ley de Cogestión de 1976), en Inglaterra y Francia esto se reduce a las empresas públicas (Ojeda 1978).

Por último, se definieron tres grupos sociales: medios de comunicación, futuras generaciones como representantes de la comunidad y grupos feministas y/o asociaciones de mujeres, que en nuestro estudio sustituyen a los grupos medioambientalistas que fueron utilizados en los trabajos citados (Díez et al., 2008; Fernández y Nieto, 2004).

Como resumen, los diez grupos de interés definidos fueron: gobierno; equipo de dirección; personal de la empresa; propietarios/as, accionistas; clientes, medios de comunicación, proveedores, sindicatos, futuras generaciones y por último, grupos feministas y asociaciones de mujeres.

El cuestionario fue enviado a las empresas españolas (sociedades anónimas) con un tamaño igual o superior a los 250 empleados. Estas empresas están obligadas por la Ley Orgánica de Igualdad de Oportunidades entre Mujeres y Hombres a realizar un Plan de Igualdad, por lo que suponemos que están más sensibilizadas con estos temas. De acuerdo con la base de datos SABI (Sistema de Análisis de Balances Ibéricos), la población objeto de estudio estaba compuesta por 2.389 empresas. Respondieron 92 empresas, lo que nos da una tasa de respuesta del 3,85\%. Esta baja tasa de respuesta es una de las conocidas desventajas de la encuesta por correo (Díaz de Rada, 2005). Sin embargo hay que decir que los datos de la muestra no presentan una situación promedio muy desviada respecto del promedio de los datos de las empresas españolas, considerando tamaño y edad así como los datos relativos a género. La tabla 1 presenta la ficha técnica de la investigación.

Tabla 1

Ficha técnica de la investigación

\begin{tabular}{ll}
\hline Población & 2.389 Empresas de mas de 250 trabajadores \\
Ámbito geográfico & España \\
Recogida de datos & Encuesta por correo Mayo/Julio 2009 \\
Encuestado & Director/a de recursos humanos \\
Tasa de respuesta & $3,85 \%$ \\
Error muestral & $10,2 \%$ \\
Nivel de confianza & $95 \%$ \\
\hline
\end{tabular}


A la hora de analizar los datos, utilizamos como variables de control, la edad de la empresa y el tamaño, medido por el número (ln) de trabajadores en plantilla. También se utilizó el sexo de la persona que respondió la encuesta en cada empresa, debido a la influencia que ser hombre o mujer puede tener en sus percepciones (Scholem y Mavondo, 2003). En cuanto a los datos relativos al género, se crearon tres categorías en función del porcentaje de hombres y mujeres en tres niveles: el equipo directivo, en el consejo de administración y la plantilla en general. Se categorizaron como "presencia equilibrada" cuando había más del $40 \%$ y menos del $60 \%$ de cualquiera de los sexos; masculinizado cuando la presencia de hombres superaba al $60 \%$ y feminizado cuando la presencia de mujeres superaba el $60 \%$. También se obtuvo información sobre su participación activa en acciones relacionadas con la Ley de Igualdad de Oportunidades entre Mujeres y Hombres. Concretamente se les pidió información sobre si habían elaborado el "plan de igualdad" preceptivo para empresas de más de 250 trabajadores y si habían incluido algún informe sobre igualdad de oportunidades entre mujeres y hombres en sus memorias anuales.

\subsection{Resultados}

La tabla 2 nos muestra los datos descriptivos de las empresas de la muestra. La mayoría de las empresas $(68,1 \%)$ tienen una antigüedad superior a 20 años y el tamaño medio de la plantilla es de 1.112 personas. El 29,3\% de las empresas tienen una presencia equilibrada de hombres y mujeres en sus plantillas, mientras que este porcentaje se reduce al $18,4 \%$ en los equipos de dirección y al $9 \%$ en los Consejos de Administración. Más de la mitad de las empresas $(55,5 \%)$ tienen una plantilla masculinizada frente al $14,1 \%$ de las empresas con una plantilla feminizada. El 81,6\% de los equipos de dirección están masculinizados así como el $91 \%$ de los Consejos de Administración. No hubo ningún caso en el que el equipo de dirección o el Consejo de Administración estuvieran feminizados.

Tabla 2

\section{Datos descriptivos de la muestra}

\begin{tabular}{llr}
\hline EDAD & Menos de 10 años & $7,7 \%$ \\
& Entre 10-20 años & $24,2 \%$ \\
& Más de 20 años & $68,1 \%$ \\
& Media: 30 años; desviación: 18; Mínima:7; Máxima: 92 & \\
\hline PLANTILLA & Media: 1.112 personas; Desviación: 2.383 & \\
\hline & Relativos al género & $29,3 \%$ \\
\hline PERSONAL & Presencia equilibrada (40-60) & $55,5 \%$ \\
& Masculinizado & $14,1 \%$ \\
& Feminizado & $36,4 \%$ \\
\hline
\end{tabular}




\begin{tabular}{llc}
\hline EQUIPO DE & Presencia equilibrada (40-60) & $18,4 \%$ \\
DIRECCIÓN & Masculinizado & $81,6 \%$ \\
& Feminizado & $0 \%$ \\
& $\%$ cargos directivos ocupados por mujeres & $18,9 \%$ \\
\hline CONSEJO DE & Presencia equilibrada (40-60) & $9,0 \%$ \\
ADMINISTRACIÓN & Masculinizado & $91,0 \%$ \\
& Feminizado & $0 \%$ \\
& $\%$ cargos en el Consejo de Adm. ocupados por mujeres & $10,5 \%$ \\
\hline Empresas que han elaborado un "plan de igualdad" & $58,2 \%$ \\
\hline Empresas que han incluido algún informe sobre igualdad de oportunidades & $43,5 \%$ \\
entre mujeres y hombres en sus memorias & $52,2 \%$ mujeres \\
\hline Sexo de la persona encuestada & $42,4 \%$ hombres \\
& $5,4 \%$ n/d \\
\hline $\mathrm{N}=92$ & \\
\hline
\end{tabular}

El 58,2\% de las empresas han elaborado un Plan de Igualdad y el 43,5\% han incluido algún informe sobre igualdad de oportunidades entre mujeres y hombres en sus memorias. Tan sólo cuatro empresas de la muestra cotizaban en bolsa. Las cuatro empresas declararon haber elaborado un Plan de Igualdad, pero tan sólo tres habían incluido algún informe sobre igualdad de oportunidades en sus memorias, lo que podría resultar sorprendente, ya que el Código Conthe obliga a las empresas cotizadas a explicar los motivos de la escasez de consejeras. Aunque esta obligación no siempre se ha cumplido y las empresas tienden a no incluir estos informes en sus memorias, sobre todo si los datos no reflejan una evolución positiva que pueda ayudar a mejorar su imagen corporativa (Grosser y Moon, 2008).

El 52,2\% de los directivos que respondieron la encuesta fueron mujeres. El alto porcentaje puede deberse a dos razones: a) las mujeres están más predispuestas a colaborar en este estudio o b) la encuesta se dirigió a la Dirección de RRHH, área en la que se concentran un elevado número de mujeres (De Luis et al., 2007).

\subsubsection{Importancia percibida de los stakeholders}

La tabla 3 nos muestra la valoración y jerarquización de la importancia percibida de los distintos stakeholders en función de la variable utilizada: presencia equilibrada en el Consejo de Administración. Los grupos organizacionales, que en el trabajo original de Agle et al. (1999) eran percibidos con mayor importancia, obtienen distintas ponderaciones en la ordenación. Los propietarios/as y accionistas son percibidos como el grupo de mayor importancia, seguido del equipo de dirección mientras que los proveedores se perciben como el grupo menos importante, resultado que coincide con el obtenido por Fernández y Nieto (2004) en relación con el medioambiente. El resto de grupos organizaciones (personal de la empresa y clientes) se entremezclan en la ordenación con los dos grupos normativos, Gobierno y sindicatos. Concretamente aparece el Gobierno en tercer lugar, el personal de la 
empresa y los sindicatos en cuarto y quinto y los clientes en sexto lugar. Por último y antes de los proveedores se sitúan los grupos sociales: futuras generaciones, medios de comunicación y grupos feministas y/o asociaciones de mujeres. La diferencia fundamental en los resultados obtenidos por Fernández y Nieto (2004) se da en la ordenación del Gobierno. En el estudio medioambiental, el Gobierno se sitúa como el grupo de mayor importancia, por delante incluso de los propietarios. La razón puede deberse a que la normativa sobre Igualdad de Oportunidades en España es más reciente que la normativa sobre Medio Ambiente y las empresas no se sienten presionadas en este tema. No obstante, es de destacar que el Gobierno se percibe como más importante que el personal de la empresa, lo que podría indicar que la consecución de una presencia equilibrada representa más un objetivo político que una demanda real de las/os trabajadoras/es por conseguir una igualdad real de oportunidades.

La tabla 3 también muestra los valores medios obtenidos para cada stakeholder en importancia y para cada uno de sus atributos. Para algunos atributos el orden de priorización cambia con respecto a la importancia dada. Es destacable el caso de los clientes, a los que se les otorga cierta importancia (sexto lugar) que no se corresponde con las puntuaciones que obtienen en los atributos de legitimidad y urgencia, que los posicionan en penúltimo lugar por delante de los proveedores, o en el atributo de poder que los posiciona con menor importancia que las futuras generaciones.

Por el contrario, a otros stakeholders con menor importancia se les reconocen altas puntuaciones en algunos atributos. Por ejemplo, a las futuras generaciones se les reconoce un poder y legitimidad superior a los empleados, sindicatos o clientes.

En cuanto a los grupos de mujeres, se puede observar que alcanzan una considerable puntuación en cuanto a legitimidad, pero se les reconoce escaso poder. En general, la jerarquización obtenida puede llevarnos a considerar adecuada la pirámide de Responsabilidad Social Corporativa propuesta por Carroll (Carroll, 1991; Carroll y Shabana, 2010) que establece un orden de responsabilidad para las empresas. En la base las responsabilidades económicas, que explicarían la mayor importancia percibida de los propietarios, a continuación las responsabilidades legales que explicaría la importancia del Gobierno. Ambos tipos de responsabilidades explicarían la importancia de los siguientes grupos: personal de la empresa; sindicatos y clientes. Por último las responsabilidades éticas y discrecionales que explicarían la importancia de los grupos sociales: futuras generaciones; medios de comunicación y grupos feministas y/o de mujeres.

Tabla 3

Valoración de la importancia y los atributos de cada stakeholder

\begin{tabular}{lcccc}
\hline \multicolumn{1}{c}{ Stakeholders } & Poder & Legitimidad & Urgencia & Importancia \\
\hline Propietarios/as, Accionistas & 5,84 & 5,22 & 4,80 & 5,95 \\
Equipo de dirección & 4,52 & 4,71 & 4,31 & 5,44 \\
Gobierno & 4,56 & 4,35 & 4,34 & 4,87 \\
Personal de la empresa & 2,63 & 3,80 & 3,31 & 4,12 \\
\hline
\end{tabular}




\begin{tabular}{lcccc}
\hline \multicolumn{1}{c}{ Stakeholders } & Poder & Legitimidad & Urgencia & Importancia \\
\hline Sindicatos & 3,13 & 3,83 & 3,73 & 4,00 \\
Clientes & 3,00 & 3,00 & 3,02 & 3,90 \\
Futuras Generaciones & 3,59 & 3,91 & 3,17 & 3,63 \\
Medios de Comunicación & 3,00 & 3,40 & 3,23 & 3,62 \\
Grupos feministas y/o de mujeres & 2,49 & 3,75 & 3,23 & 3,05 \\
Proveedores & 2,23 & 2,54 & 2,38 & 2,94 \\
\hline
\end{tabular}

\subsubsection{Efecto individualizado}

Para contrastar H1a, H1b y H1c hemos observado el efecto individualizado de cada atributo sobre la importancia percibida. Para ello, se han calculado las correlaciones bivariadas utilizando el coeficiente de Spearman debido a la falta de normalidad de los datos disponibles (tabla 4), y se han realizado análisis de regresión lineal para cada uno de los diez grupos de interés considerados para observar el efecto conjunto de los tres atributos (tabla 5).

Tabla 4

Correlación de los atributos con la importancia percibida de cada stakeholder

\begin{tabular}{lccc}
\hline \multicolumn{1}{c}{ Rho de Spearman } & Poder & Legitimidad & Urgencia \\
\hline Propietarios/as, Accionistas & $0,271^{*}$ & $0,434 * *$ & $0,414^{* *}$ \\
Equipo de dirección & $0,428^{* *}$ & $0,394^{* *}$ & $0,579^{* *}$ \\
Gobierno & $0,515^{* *}$ & $0,474 * *$ & $0,608^{* *}$ \\
Personal de la empresa & $0,465^{* *}$ & $0,547 * *$ & $0,608^{* *}$ \\
Sindicatos & $0,665^{* *}$ & $0,629 * *$ & $0,772^{* *}$ \\
Clientes & $0,485^{* *}$ & $0,501 * *$ & $0,698^{* *}$ \\
Futuras generaciones & $0,573^{* *}$ & $0,625^{* *}$ & $0,664^{* *}$ \\
Medios de comunicación & $0,593^{* *}$ & $0,611^{* *}$ & $0,764 * *$ \\
Grupos feministas y/o de mujeres & $0,640^{* *}$ & $0,546^{* *}$ & $0,694 * *$ \\
Proveedores & $0,581^{* *}$ & $0,680^{* *}$ & $0,693 * *$ \\
\hline
\end{tabular}

** La correlación es significativa al nivel 0,01; * la correlación es significativa al nivel 0,05.

La tabla 4 nos muestra las correlaciones bivariadas de cada uno de los atributos con la importancia percibida. La correlación es positiva y significativa para cada uno de los atributos en todos los stakeholders, lo que confirma las hipótesis planteadas. 
Tabla 5

Resultados de la regresión lineal

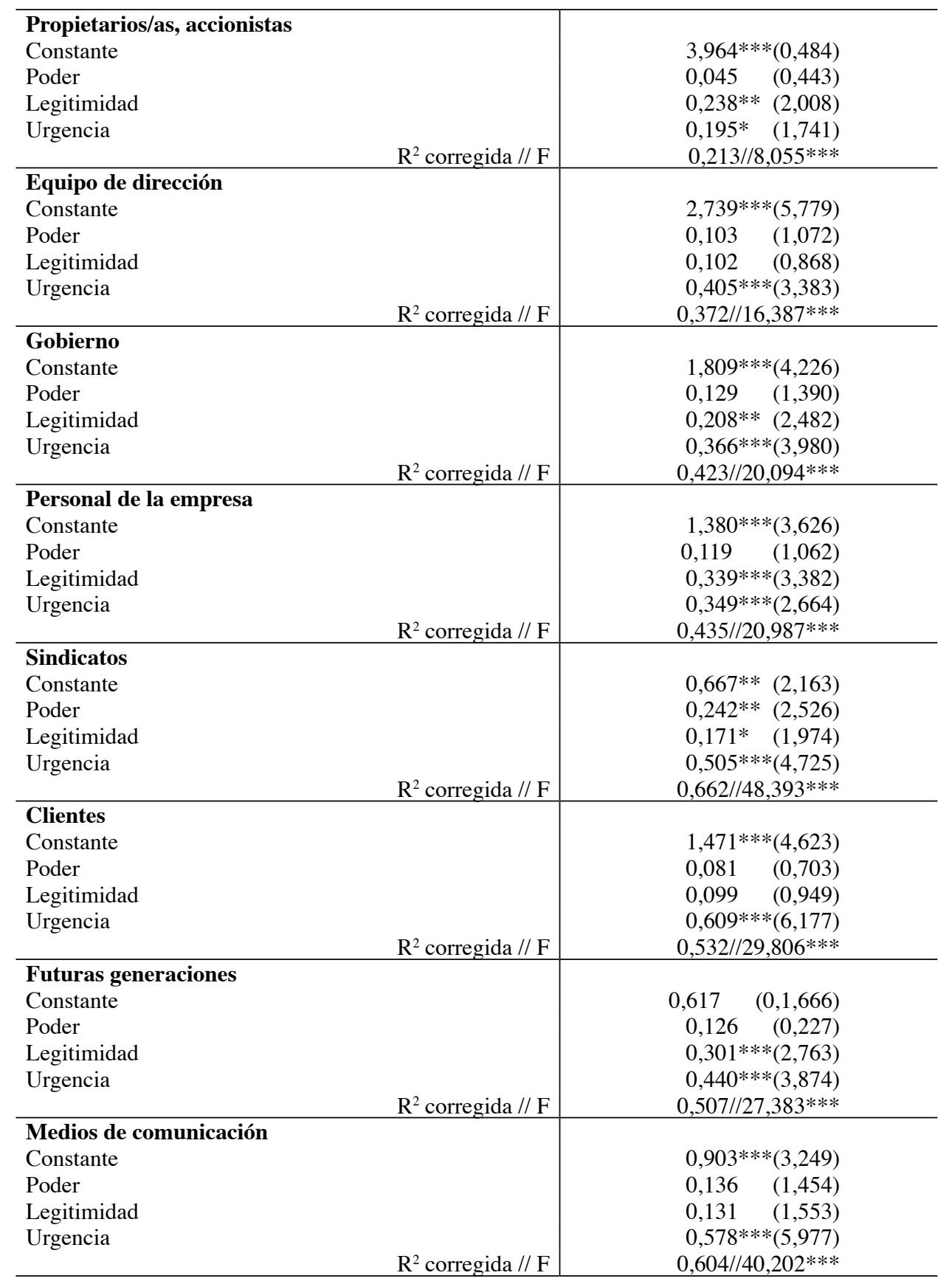




\begin{tabular}{|c|c|}
\hline $\begin{array}{l}\text { Grupos feministas y/o de mujeres } \\
\text { Constante } \\
\text { Poder } \\
\text { Legitimidad } \\
\text { Urgencia }\end{array}$ & $\begin{array}{rr}0,302 & (1,019) \\
0,365 * * *(3,677) \\
0,142 * \quad(1,826) \\
0,404 * * *(4,745) \\
0,603 / / 40,023 * * * \\
\end{array}$ \\
\hline $\begin{array}{l}\text { Proveedores } \\
\text { Constante } \\
\text { Poder } \\
\text { Legitimidad } \\
\text { Urgencia }\end{array}$ & $\begin{array}{cr}1,069 * * *(3,987) \\
0,073 & (0,554) \\
0,213 * & (1,742) \\
0,508 * * *(3,740) \\
0,501 / / 26,748 * * *\end{array}$ \\
\hline
\end{tabular}

Variable dependiente: Importancia percibida de cada stakeholder. $* \mathrm{p}<0,1 * * \mathrm{p}<0,05 ; * * * \mathrm{p}<0,01$. Error estándar entre paréntesis.

La tabla 5 presenta los resultados obtenidos en el análisis de regresión que se realizó para observar la influencia conjunta de los tres atributos sobre la importancia percibida, de forma que podemos comprobar cuál o cuáles de los atributos resultan ser más influyentes para determinar la importancia de cada stakeholder. Las regresiones obtenidas presentan unos $\mathrm{R}^{2}$ corregidos que varían desde un valor de 0,213 para la importancia de los propietarios a un valor de 0,662 para los sindicatos. En todos los casos la relación observada es positiva. Para el equipo de dirección y los clientes, es la urgencia el atributo influyente. Legitimidad y urgencia son los atributos que explican la importancia percibida de los propietarios, el Gobierno, empleados, generaciones futuras, medios de comunicación y proveedores. En el caso de los sindicatos y grupos de mujeres, los resultados son muy similares, los tres atributos explican la importancia percibida siendo la legitimidad el atributo de menor impacto. En general, se observa que la urgencia explica la importancia en todos los grupos, la legitimidad la explica en siete de los diez grupos, mientras que el poder, solo lo hace en dos de los diez grupos: los sindicatos y los grupos de mujeres.

\subsubsection{Efecto acumulativo}

Para verificar la H1d se han clasificado los stakeholders en función del número de atributos de poseen, dando lugar a cuatro clasificaciones, si no poseen ningún atributo, o bien poseen, uno, dos o tres atributos. Para determinar si un stakeholder posee o no un atributo se ha utilizado el método propuesto por Agle et al. (1999) según el cual se considera que un stakeholder posee un atributo si se encuentra por encima de la valoración media de ese atributo para el total de la muestra.

Para ello realizamos dos tipos de análisis (tabla 6). En primer lugar utilizamos la prueba de Kruskal-Wallis para contrastar la hipótesis nula de que varias muestras provienen de la misma población y, por tanto, tienen igualdad de promedios poblacionales. En segundo lugar calculamos la correlación (Rho de Spearman) para observar cuál era el sentido de dicha relación. La prueba de Kruskal-Wallis nos confirmó que existen diferencias en los promedios poblacionales respecto a los atributos acumulados en todos los stakeholders, 
con lo que se confirma que existen diferencias significativas entre los distintos niveles de importancia percibida por los directivos y el número de atributos que poseen. El coeficiente de correlación nos indica que el sentido de la relación es positivo en todos los casos. Con lo que se confirma la hipótesis H1d planteada.

Tabla 6

Relación entre el número de atributos que poseen y la importancia percibida

\begin{tabular}{|c|c|c|c|c|}
\hline Stakeholders & $\begin{array}{c}\text { Kruskal- } \\
\text { Wallis }\end{array}$ & Correlación & $\begin{array}{c}\text { Kruskal- } \\
\text { Wallis }\end{array}$ & Correlación \\
\hline & Chi-cuadrado & $\begin{array}{l}\text { Rho de } \\
\text { Spearman }\end{array}$ & Chi-cuadrado & $\begin{array}{c}\text { Rho de } \\
\text { Spearman }\end{array}$ \\
\hline Propietarios/as, accionistas & $13,587 * *$ & $0,395 * * *$ & $11,286^{*}$ & $0,356 * * *$ \\
\hline Equipo de dirección & $21,566 * * *$ & $0,450 * * *$ & $18,736^{* * *}$ & $0,444 * * *$ \\
\hline Gobierno & $33,694 * * *$ & $0,609 * * *$ & $29,733 * * *$ & $0,610 * * *$ \\
\hline Personal de la empresa & $33,137 * * *$ & $0,604 * * *$ & $26,969 * * *$ & $0,549 * * *$ \\
\hline Sindicatos & $42,151 * * *$ & $0,696 * * *$ & $37,166^{* * *}$ & $0,684 * * *$ \\
\hline Clientes & $32,331 * * *$ & $0,597 * * *$ & $29,255^{* * *}$ & $0,620 * * *$ \\
\hline Futuras generaciones & $44,674 * * *$ & $0,723 * * *$ & $33,221 * * *$ & $0,636^{* * *}$ \\
\hline Medios de comunicación & $43,548 * * *$ & $0,711^{* * *}$ & $40,649 * * *$ & $0,689 * * *$ \\
\hline Grupos feministas y/o de mujeres & $45,565 * * *$ & $0,731 * * *$ & $36,069 * * *$ & $0,672 * * *$ \\
\hline Proveedores & $37,647 * * *$ & $0,659 * * *$ & $39,770 * * *$ & $0,701 * * *$ \\
\hline
\end{tabular}

* $\mathrm{p}<0,01 * * \mathrm{p}<0,005 ; * * * \mathrm{p}<0,001$

\subsubsection{Efecto género}

El análisis de regresión presentado en la tabla 5 se repitió incluyendo diversas variables de control. En concreto se incluyeron las siguientes variables:

1. Número de trabajadores en plantilla $(\ln )$

2. Edad de la empresa medida en años

3. Grado de sexualización de la plantilla. Se crearon tres variables: Plantilla masculinizada; Plantilla feminizada y Plantilla con presencia equilibrada; que tomaban valores 0,1 .

4. Grado de sexualización del equipo de dirección. Se crearon dos variables: Dirección masculinizada y Dirección con presencia equilibrada, ya que no existía ninguna empresa con dirección feminizada, que tomaban valores 0,1 .

5. Grado de sexualización del Consejo de Administración. Se crearon dos variables: Consejo masculinizado y Consejo con presencia equilibrada, ya que no existía ninguna empresa con Consejo feminizado, que tomaban valores 0,1 .

6. Plan de Igualdad, que tomaba el valor 1, si la empresa había declarado que habían elaborado un Plan de Igualdad. 
7. Igualdad en la Memoria, que tomaba el valor 1, si la empresa había declarado que habían incluido algún informe sobre Igualdad en la Memoria

8. Sexo de la persona que contestó a la encuesta, que tomaba el valor 0 si era hombre o el valor 1 si era mujer.

Dado el elevado número de variables explicativas, se utilizó la regresión por pasos, de forma que tan sólo las variables significativas fueran incluidas en la solución. Los resultados de estas regresiones fueron similares a los obtenidos en la tabla 5, con una excepción: la regresión referida a la importancia de los grupos feministas y/o de mujeres. Para el resto de stakeholders, ninguna de las nuevas variables introducidas resultó significativa.

Tabla 7

\section{Resultados de la regresión lineal}

\section{Grupos feministas y/o de mujeres}

\begin{tabular}{|c|c|}
\hline Constante & $0,289 \quad(0,341)$ \\
\hline Urgencia & $0,498 * * *(0,083)$ \\
\hline Poder & $0,256^{* *} \quad(0,110)$ \\
\hline Plantilla feminizada & $-1,157 * *(0,448)$ \\
\hline Sexo de la persona encuestada (mujer) & $0,617 * *(0,283)$ \\
\hline Plan de igualdad & $0,615 * *(0,285)$ \\
\hline $\mathrm{R}^{2}$ corregida // $\mathrm{F}$ & $0,637 / / 24,125^{* * *}$ \\
\hline
\end{tabular}

Variable dependiente: Importancia percibida de los grupos feministas y/o de mujeres. ${ }^{*} \mathrm{p}<0,1 * *^{*} \mathrm{p}<0,05$; $* * * \mathrm{p}<0,01$. Error estándar entre paréntesis.

Al introducir las nuevas variables, el valor explicativo del modelo aumenta de un $\mathrm{R}^{2}$ de 0,603 a 0,637 (tabla 7). La urgencia y el poder se mantienen como atributos explicativos, pero la legitimidad deja de ser significativa. Tres variables relativas al género resultan significativas para explicar la importancia del grupo. Que la empresa haya realizado un Plan de Igualdad influye positivamente y que la plantilla esté feminizada influye negativamente. Por último, ser mujer influye positivamente a la hora de valorar la importancia de este grupo. Existen antecedentes de que las mujeres tienden a dar valoraciones más altas que los hombres a los stakeholders, aunque no se han encontrado diferencias en la jerarquización de los grupos (Scholem y Mavondo, 2003). La pregunta que nos hicimos fue si las directivas de esta muestra de empresas españolas daban una mayor importancia a los grupos feministas o este fenómeno de puntuar con mayor intensidad era común a otros stakaholders. Para comprobarlo, se realizó un análisis ANOVA en el que se pudo observar que había diferencias significativas en las puntuaciones que hombres y mujeres daban a siete de los diez stakeholders considerados y en todos los casos las puntuaciones de las mujeres eran superiores a las de los hombres. También se observó que, al igual que en el estudio de Scholem y Mavondo (2003), la diferente puntuación no cambiaba el orden de importancia de los grupos (tabla 8). 
Tabla 8

Valoración de la importancia y los atributos de cada stakeholder, en función del sexo de la persona que responde a la encuesta: hombre $(\mathrm{H})$ o mujer $(\mathrm{M})$

\begin{tabular}{|l|c|c|c|c|c|c|c|c|}
\hline \multicolumn{1}{|c|}{ Stakeholders } & \multicolumn{2}{c|}{ Poder } & \multicolumn{2}{c|}{ Legitimidad } & \multicolumn{2}{c|}{ Urgencia } & \multicolumn{2}{c|}{ Importancia } \\
\hline & $\mathrm{H}$ & $\mathrm{M}$ & $\mathrm{H}$ & $\mathrm{M}$ & $\mathrm{H}$ & $\mathrm{M}$ & $\mathrm{H}$ & $\mathrm{M}$ \\
\hline $\begin{array}{l}\text { Propietarios/as, } \\
\text { Accionistas }\end{array}$ & 5,58 & 5,93 & 5,37 & 5,26 & 4,83 & 4,90 & 5,85 & 6,07 \\
\hline Equipo de dirección & 3,57 & $5,37 * * *$ & 4,58 & 4,93 & 3,89 & $4,79 * *$ & 4,97 & $5,90 * *$ \\
\hline Gobierno & 4,58 & 4,56 & 4,14 & 4,60 & 4,17 & 4,57 & 4,77 & 5,19 \\
\hline Personal de la empresa & 2,11 & $3,15 * * *$ & 3,39 & $4,29 * *$ & 2,94 & $3,76 * *$ & 3,63 & $4,67 * *$ \\
\hline Sindicatos & 2,59 & $3,83^{* * *}$ & 3,60 & 4,19 & 3,11 & 4,37 & 3,57 & $4,59 * *$ \\
\hline Clientes & 2,76 & 3,28 & 2,83 & 3,24 & 2,83 & 3,32 & 3,57 & $4,29 *$ \\
\hline Futuras Generaciones & 3,24 & $4,03 *$ & 3,71 & 4,22 & 2.78 & $3,52 *$ & 3,14 & $4,12 * *$ \\
\hline Medios de Comunicación & 2,42 & $3,61 * * *$ & 3,31 & 3,55 & 2,78 & $3,67 * *$ & 3,17 & $4,14 * * *$ \\
\hline $\begin{array}{l}\text { Grupos feministas y/o de } \\
\text { mujeres }\end{array}$ & 2,24 & $2,83 *$ & 3,89 & 3,83 & 3,03 & 3,56 & 2,60 & $3,59 * *$ \\
\hline Proveedores & 1,92 & $2,50 *$ & 2,54 & 2,59 & 2,25 & 2,54 & 2,74 & 3,14 \\
\hline
\end{tabular}

$* \mathrm{p}<0,1 * \mathrm{p}<0,05 ; * * * \mathrm{p}<0,01$

Se realizó un ANOVA para ver la influencia del resto de variables relativas al género y se encontraron algunas diferencias significativas. Las empresas que habían elaborado el Plan de Igualdad e incluido informe de género en sus memorias valoraban con mayor intensidad a los medios de comunicación y a los grupos feministas. Haber elaborado un Plan de Igualdad también era influyente para valorar con mayor intensidad la importancia del Gobierno. La existencia o no de presencia equilibrada en la empresa no influían significativamente en ningún caso.

\section{CONCLUSIONES}

El principio de presencia equilibrada en los Consejos de Administración en las empresas está siendo objeto de una fuerte demanda social habiendo llegado en España a ser objeto de regulación jurídica. La revisión de la literatura nos lleva a pensar que las empresas asumirán un comportamiento proactivo para conseguir la presencia equilibrada, en la medida en que perciban presión de los grupos de interés. Este artículo presenta un estudio cuyo objetivo es ayudar a reconocer qué stakeholders perciben las empresas como más importantes en relación al tema considerado. Para ello, se ha replicado el modelo propuesto por Mitchell et al. (1997) y que fue ampliado por Agle et al. (1999). El modelo propone que las demandas de los stakeholders serán atendidas en la medida en que los/as directivos/as 
los perciban importantes y que esa importancia percibida estará a su vez condicionada por la percepción que tengan sobre tres atributos en cada uno de los grupos de interés considerados: poder para presionar, legitimidad de sus demandas y urgencia en la respuesta que esperan de la empresa. Los resultados de este estudio sugieren que esta relación existe para todos los stakeholder considerados, así como que una mayor acumulación de atributos, aumenta la importancia percibida, sin embargo, la intensidad en que los atributos influyen para valorar la importancia varía entre los grupos.

Los resultados hacen pensar que los propietarios son el grupo de mayor importancia y también el primero en cuanto a la valoración de los atributos considerados, es decir, el grupo con mayor poder, con mayor legitimidad y que demandan respuesta con mayor urgencia, lo que nos muestra que los objetivos económicos siguen siendo prioritarios en las empresas. Pero también se ha observado que el Gobierno se percibe como muy importante, más que otros grupos organizacionales, como el personal de las empresa, los clientes y los proveedores. A pesar de que se le reconoce un gran poder al Gobierno, son la legitimidad y urgencia los atributos que explican su importancia percibida, lo que nos hace dudar de la efectividad que tendría el desarrollo de una normativa obligatoria, más allá de la recomendación actual.

Dado que estamos defendiendo una mayor diversidad de género en los Consejos de Administración, merece la pena destacar que, de acuerdo con los resultados de este estudio, una mayor presencia de mujeres en la cúpula empresarial podría aumentar la intensidad de la importancia percibida de los stakeholders, lo que podría favorecer un mayor desarrollo de los informes de Responsabilidad Social Corporativa, pero no el orden de importancia de los grupos, por lo que el orden de importancia de responsabilidades propuesto por Carroll se mantendría invariable: económicas, legales, éticas y por último discrecionales (Carroll, 1991; Carroll y Shabana, 2010).

Las variables relativas al género sólo resultan influyentes para determinar la importancia de uno de los grupos de interés: los grupos feministas y/o de mujeres. Que la persona que opina en la Dirección sea una mujer y que la empresa haya puesto en marcha el Plan de Igualdad influye positivamente en la importancia percibida de este grupo, mientras que tener una plantilla feminizada (más del 60\%) influye negativamente.

Los resultados sugieren futuras líneas de investigación. Una vez determinados cuáles son los stakeholders más relevantes percibidos por la dirección de la empresa en el tema de presencia equilibrada, se debería profundizar en conocer con más exactitud cuáles son las demandas reales de estos grupos, al margen de la percepción de los directivos tengan de la mayor o menor importancia de cada uno de ellos, para diseñar una política de Responsabilidad Social Corporativa que pueda darles respuesta, o comprobar si las medidas ya existentes se ajustan a estas demandas, puesto que en el medio y largo plazo, el objetivo financiero de la empresa no es en absoluto independiente del objetivo de equidad social. De acuerdo a ello y según nuestros resultados, la Responsabilidad Social Corporativa de las empresas encaminada a la consecución de la presencia equilibrada en los consejos de administración, debería de analizar y tener en cuenta realmente cuáles son las demandas no sólo de los accionistas, equipo de dirección o gobierno, sino también las del personal, sindicatos, clientes, futuras generaciones, medios de comunicación, grupos de mujeres y proveedores, al margen de la mayor o menor importancia percibida por los directivos y del poder que estos grupos puedan tener para exigir sus demandas. 
Este estudio presenta ciertas limitaciones. En primer lugar una baja tasa de respuesta, debida a la recogida de datos por correo, que puede contener sesgos en los resultados. En segundo lugar, los datos se basan en la percepción de una única persona de la Dirección en cada empresa. Por último, se ha partido de una clasificación de stakeholders que ha resultado útil para el estudio de otros temas como el medioambiente, que a menudo son considerados como "sociales" y que comparten el objetivo de sostenibilidad, pero la trascendencia de un tema relativo a la igualdad de oportunidades entre mujeres y hombres que afecta a muchas decisiones vitales, dentro y fuera del mercado, podría provocar que nuevos grupos de interés no considerados hasta ahora.

\section{BIBLIOGRAFÍA}

ADLER, R. (2001): "Women in the executive suite correlate to high profits", http://glassceiling.org/ InTheNewsFolder/HBRArticlePrintablePage.html.

AGLE, B.R.; MITCHELL, R.K. y SONNENFELD, J.A. (1999): "Who Matters to CEOS? An Investigation of Stakeholder Attributes and Salience, Corporate Performance, and CEO Values", Academy of Management Journal, Vol. 42, №. 5, pp. 507-525.

BILIMORIA, D. (2006): "The Relationship between Women Corporate Directors and Women Corporate Officers", Journal of Managerial Issues, Vol. 13, N. 1, pp. 47-61.

BRAMMER, S., MILLINGTON, A. y PAVELIN, S. (2009): "Corporate Reputation and Women on the Board" British Journal of Management, Vol. 20, No. 1, pp 17-29.

BURTON, B. K. y DUNN, C.P. (1996):"Feminist Ethics as Moral Grounding for Stakeholder Theory" Business Ethics Quarterly, Vol. 6, №. 2, pp. 133-147.

CARRASCO, A. y LAFFARGA, J. (2007) "La diversidad de género en el CODIG Unificado español y la práctica empresarial", Pecunia, $\mathrm{N}^{\circ}$. 4, pp. 1-25.

CARROLL, A.B. (1991): "The Pyramid of Corporate Social Responsibility: toward the Moral Management of Organizational Stakeholders", Business Horizons, Vol. 34, №. 4, pp. 39-48.

CARROLL, A.B. y SHABANA, K.M. (2010): "The Business Case for Corporate Social Responsibility: A Review of Concepts, Research and Practice", International Journal of Management Reviews, DOI: 10.1111/j.1468-2370.2009.00275.x

CARTER, D.; SIMKINS, B. y SIMPSON, W. (2003): "Corporate Governance, Board Diversity, and Firm Value", the Financial Review, Vol. 38, №. 1, pp. 33-53.

CRAGG, W. (2002): "Business Ethics and Stakeholder Theory", Business Ethics Quarterly, Vol. 12 $N^{\circ} .2$, pp. 113-142.

CRITTENDEN, C. (2000): "Ecofeminism Meets Business: A Comparison Of Ecofeminist, Corporate, and Free Market Ideologies", Journal of Business Ethics, Vol. 24 №. 1, pp. 51-63.

DE LUIS, P.; MARTINEZ, A.; PEREZ, M. y VELA, M.J. (2007): "La diversidad de género en la alta dirección de las mayores empresas españolas", Investigaciones Europeas de Dirección y Economía de la Empresa, Vol. 13, N. 2 pp. 33-53.

DEL BRIO, E. y DEL BRIO, I. (2009): “Los Consejos de Administración en las Sociedades Cotizadas: Avanzando en Femenino", Revista de Estudios Empresariales, №.1, pp. 102-118.

DIAZ DE RADA, V. (2005): "Measure and control of non-response in a mail survey", European Journal of Marketing, Vol. 39, No. 1-2, pp. 16-32.

DÍEZ, F.; MEDRANO, M. L. y DÍEZ, E. P. (2008): "Los grupos de interés y la presión medioambiental”, Revista Cuadernos de Gestión, Vol.8; №.2, pp. 81-96.

DIMOWSKI, W. y BROOKS, R. (2008): “The gender composition of boards after an IPO”, Corporate Governance, Vol. 6, № 1, pp 11-17. 
DU RIETZ, A. y HENREKSON, M. (2000): “Testing the female underperformance hypothesis, Small Business Economics", Vol. 14, №. 1, pp. 1-10.

ELÓSEGUI, M y LOUSADA, F. (2007): "La participación de las mujeres en los consejos de administración de las sociedades mercantiles", Revista del Poder Judicial, No. 86, pp. 255-284.

ELY, R. y PADAVIC, I. (2007): "A feminist Analysis of Organizational Research on Sex Differences", Academic of Management Review, Vol. 32, No. 4, pp. 1121-1143.

ERHARDT, N., WERBEL, J. and SCHRADER, C. (2003): Board of director diversity and firm financial performance, Corporate Governance: An International Review, Vol.11, №. 2 , pp. 102-111.

EUROPEAN COMMISSION (2004): Green Paper: Equality and non-discrimination in an Enlarged European Union: Fundamental Rights and Anti-discrimination, Luxemburgo, Office for Official Publications of the European Communities.

EUROPEAN COMMISSION (2005): Work Programme for 2005, for the Implementation of the Framework Strategy on Gender Equality, European Commission. SEC (2005): 1044, Brussels.

FERNANDEZ, R. y NIETO M. (2004): "Stakeholder Salience in Corporate Environmental Strategy”, Corporate Governance, Vol. 4, №. 3, pp. 65-76.

FRANCOEUR, C.; LABELLE, R. y SINCLAIR-DESGAGNÉ, B. (2007): "Gender Diversity in Corporate Governance and Top Management”, Journal of Business Ethics, Vol. 81, №. 1, pp. 83-95.

FREEMAN, R.E. (1984): Strategic Management: A Stakeholder Approach, Boston, Pitman.

GALBREATH, J.: "How Does Corporate Social Responsibility Benefit Firms? Evidence From Australia, Curtin University Of Technology, Perth, Australia.

GREENWOOD, M. (2007): "Stakeholder Engagement: Beyond the Myth of Corporate Responsibility", Journal of Business Ethics, Vol. 74, №. 4, pp. 315-327.

GROSSER, K y MOON, J (2005): "Gender Mainstreaming and Corporate Social Responsibility: Reporting Workplace Issues”, Journal of Business Ethics, Vol. 62, №. 4, pp. 327-340.

GROSSER, K y MOON, J (2008): "Developments in company reporting on workplace gender equality? A corporate social responsibility perspective", Accounting Forum, Vol. 32, No. 3, pp. 179198.

GROSSER, K. (2009): "Corporate social responsibility and gender equality: women as stakeholders and the European Union sustainability strategy", Business Ethics: A European Review, Vol. 18, No. 3, pp. 290-307.

HAUSMANN, R.; TYSON, L. D. y ZAHIDI, S. (2010): “The Global Gender Gap Report” World Economic Forum.

HENRIQUES, I. y SADORSKY, R.F. (1999): "Stakeholders, Social Responsibility and Performance: Empirical Evidence and Theoretical Perspectives", Academy of Management Journal, Vol. 42, No. 5, pp. 479-487.

HUSE, M. y SOLVERG, A.G. (2006): "Gender Related Boardroom Dynamics: How Scandinavian Women Make and Can Make Contributions on Corporate Boards", Women in Management Review, Vol. 21, №. 2, pp. 113-130.

INSTITUTO DE LA MUJER (2011): Estadísticas "Poder y toma de decisiones" www.inmujer.es/ ss/Satellite

KOCHAN, T., BEZRUKOVA, K., ELY, R., JACKSON, S., JOSHI, A., JEHN, K., LEONARD, J., LEVINE, D. y THOMAS, D. (2003): "The effects of diversity on business performance: report of the diversity network", Human Resource Management, Vol. 42, №.1 pp. 3-21.

KRISHNAN, H. y PARK, D. (2005): "A few good women on top management teams", Journal of Business Research, Vol. 58, No. 12, pp. 1712-1720.

LAPLUME, A.O.; SONPAR, K. y LITZ, R.A.: (2008): "Stakeholder theory: Reviewing a theory that moves us", Journal of Management, Vol. 34, No. 6, pp. 1152-1189. 
MACHOLD, S; AHMED, P. K., y FARQUHAR, S.S. (2008): "Corporate Governance and Ethics: A Feminist Perspective", Journal of Business Ethics, Vol. 81, №. 3, pp. 665-678.

MATEOS DE CABO, R, GIMENO, R, y ESCOT, L (2011): "Disentangling Discrimination on Spanish Boards of Directors", Corporate Governance: an International Review, Vol. 19, №. 1, pp. 77-95.

MITCHELL, R.K.; AGLE, B.R. y WOOD, D.J. (1997): “Toward a Theory of Stakeholder Identification and Salience: Defining the Principle of Who and What Really Counts", Academy of Management Review, Vol. 22, No.4, pp. 853-886.

OJEDA, A. (1978): La congestión de las grandes empresas en Europa: la experiencia alemana y la Ley de cogestión de 1976, Sevilla, Universidad de Sevilla

PANDEY, A. (2010), "Greening Garhwal through Stakeholder Engagement: the Role of Ecofeminism, Community and the State in Sustainable Development", Sustainable Development, Vol. $18, \mathrm{~N}^{\circ} .1, \mathrm{p} .12-19$.

REED, M.S. (2008): "Stakeholder participation for environmental management: a literature review", Biological Conservation, Vol. 141, №. 10, pp. 2417-2431.

RODRÍGUEZ, J. M. (2003): El gobierno de la Empresa: Un Enfoque Alternativo, Madrid, Ediciones Akal.

RODRÍGUEZ, J. M. (2006): "La Responsabilidad Social de la Empresa: ¿Un Medio o un Fin?”, Revista de Dirección y Administración de Empresas, №. 13, 53-77.

ROSE, C. (2007): Does female board representation influence firm performance? The Danish evidence, Corporate Governance: An International Review, Vol. 15, №. 2, 404-413.

RYAN, M. y HASLAM, S.A. (2005): "The glass cliff: evidence that women are over-represented in precarious leadership positions", British Journal of Management, Vol. 16, $\mathrm{N}^{\circ}$. 1, pp. 81-90

RYAN, M. y HASLAM, S.A. (2007): "The glass cliff: exploring the dynamics surrounding the appointment of women to precarious leadership positions", Academy of Management Review, Vol. 32, No. 2, 549-572.

SCHOLEM, P. y MAVONDO, F. (2003): Stakeholders \& Gender: A New Phenomenon?, Adelaide, ANZMAC 2003 Conference Proceedings.

SCHYNS, B. y SANDERS, K. (2005): "Exploring Gender Differences in Leaders' Occupational Self-Efficacy", Women in Management Review, Vol. 20, No. 7, pp. 513-523.

SMITH, N.; SMITH, V. y VERNER, M. (2006): "Do Women in Top Management Affect Firm Performance? A Panel Study of 2,500 Danish Firms", International Journal of Productivity and Performance Management, Vol. 55, No. 7, pp. 569-593.

SMYTH, H. (2008): “The Credibility Gap in Stakeholder Management: Ethics and Evidence of Relationship Management", Construction Management and Economics, Vol. 26 No. 6, pp. 633643.

SYED, J. y MURRAY PP. (2008): "A Cultural Feminist Approach Towards Managing Diversity in Top Management Teams", Equal Opportunities International, Vol. 27, №. 5, pp. 413-432.

VELEVA, V. (2005): Gender Diversity and Financial Performance, Citizens Advisers, Inc. http:// www.citizensfunds.org/common/pdfs/Gender_Diversity_Paper_1105.pdf

WICKS, A.; GILBERT JR. D. y FREEMAN, E. (1994): "A feminist reinterpretation of the stakeholder concept", Business Ethics Quarterly, Vol. 4, No. 4, pp. 475-498. 


\section{ANEXO I}

\section{UNIVERSIDAD DE XXXXXXXXXX}

CUESTIONARIO SOBRE LA IMPORTANCIA DE LOS STAKEHOLDERS EN LAS ESTRATEGIAS DE IGUALDAD DE OPORTUNIDADES ENTRE MUJERES Y HOMBRES

EMPRESA Teléfono

E-mail

El objetivo de este cuestionario es determinar la importancia percibida por Vds. de los diferentes grupos de interés relacionados con su empresa, ante posibles demandas para implantar estrategias que favorezcan la consecución de una "presencia equilibrada" entre mujeres y hombres en el Consejo de Administración de su empresa. La información que nos suministre será considerada confidencial y los datos únicamente se analizarán de forma agregada.

Indique por favor en qué medida percibe que cada uno de los siguientes grupos relacionados con su empresa puede utilizar (tanto si lo hace como si no), cualquier tipo de presión externa, incluso influyendo en su imagen pública, para conseguir el cumplimiento de las preocupaciones que tiene sobre la consecución de la mencionada "presencia equilibrada". Utilice una escala de 1 "ningún poder" a 7 "máximo poder":

\begin{tabular}{|l|l|l|l|l|l|l|l|}
\hline Propietarios, accionistas & 1 & 2 & 3 & 4 & 5 & 6 & 7 \\
\hline Personal de la empresa, asalariados & 1 & 2 & 3 & 4 & 5 & 6 & 7 \\
\hline Gobierno & 1 & 2 & 3 & 4 & 5 & 6 & 7 \\
\hline Clientes & 1 & 2 & 3 & 4 & 5 & 6 & 7 \\
\hline Proveedores & 1 & 2 & 3 & 4 & 5 & 6 & 7 \\
\hline Medios de comunicación & 1 & 2 & 3 & 4 & 5 & 6 & 7 \\
\hline Sindicatos & 1 & 2 & 3 & 4 & 5 & 6 & 7 \\
\hline Futuras generaciones & 1 & 2 & 3 & 4 & 5 & 6 & 7 \\
\hline Grupos feministas y/o asociaciones de mujeres & 1 & 2 & 3 & 4 & 5 & 6 & 7 \\
\hline
\end{tabular}

Indique por favor en qué medida considera que las preocupaciones de cada uno de los siguientes grupos de interés tiene sobre la obtención de una "presencia equilibrada" son consideradas adecuadas y/o legítimas Utilice una escala de 1 "ninguna legitimidad" a 7 "máxima legitimidad": 


\begin{tabular}{|l|c|c|c|c|c|c|c|}
\hline Propietarios, accionistas & 1 & 2 & 3 & 4 & 5 & 6 & 7 \\
\hline Personal de la empresa, asalariados & 1 & 2 & 3 & 4 & 5 & 6 & 7 \\
\hline Gobierno & 1 & 2 & 3 & 4 & 5 & 6 & 7 \\
\hline Clientes & 1 & 2 & 3 & 4 & 5 & 6 & 7 \\
\hline Proveedores & 1 & 2 & 3 & 4 & 5 & 6 & 7 \\
\hline Medios de comunicación & 1 & 2 & 3 & 4 & 5 & 6 & 7 \\
\hline Sindicatos & 1 & 2 & 3 & 4 & 5 & 6 & 7 \\
\hline Futuras generaciones & 1 & 2 & 3 & 4 & 5 & 6 & 7 \\
\hline Grupos feministas y/o asociaciones de mujeres & 1 & 2 & 3 & 4 & 5 & 6 & 7 \\
\hline
\end{tabular}

Indique por favor en qué medida considera que las preocupaciones de cada uno de los siguientes grupos de interés tiene sobre la obtención de una "presencia equilibrada" conlleva la exigencia de una rápida respuesta a sus demandas. Utilice una escala de 1 "ninguna urgencia" a 7 "máxima urgencia":

\begin{tabular}{|l|l|l|l|l|l|l|l|}
\hline Propietarios, accionistas & 1 & 2 & 3 & 4 & 5 & 6 & 7 \\
\hline Personal de la empresa, asalariados & 1 & 2 & 3 & 4 & 5 & 6 & 7 \\
\hline Gobierno & 1 & 2 & 3 & 4 & 5 & 6 & 7 \\
\hline Clientes & 1 & 2 & 3 & 4 & 5 & 6 & 7 \\
\hline Proveedores & 1 & 2 & 3 & 4 & 5 & 6 & 7 \\
\hline Medios de comunicación & 1 & 2 & 3 & 4 & 5 & 6 & 7 \\
\hline Sindicatos & 1 & 2 & 3 & 4 & 5 & 6 & 7 \\
\hline Futuras generaciones & 1 & 2 & 3 & 4 & 5 & 6 & 7 \\
\hline Grupos feministas y/o asociaciones de mujeres & 1 & 2 & 3 & 4 & 5 & 6 & 7 \\
\hline
\end{tabular}

Indique por favor en qué medida considera importantes a cada uno de los siguientes grupos de interés, en función del grado de atención, tiempo y prioridad que demanda sobre la obtención de una "presencia equilibrada". Utilice una escala de 1 "ninguna importancia" a 7 "máxima importancia":

\begin{tabular}{|l|l|l|l|l|l|l|l|}
\hline Propietarios, accionistas & 1 & 2 & 3 & 4 & 5 & 6 & 7 \\
\hline Personal de la empresa, asalariados & 1 & 2 & 3 & 4 & 5 & 6 & 7 \\
\hline Gobierno & 1 & 2 & 3 & 4 & 5 & 6 & 7 \\
\hline Clientes & 1 & 2 & 3 & 4 & 5 & 6 & 7 \\
\hline Proveedores & 1 & 2 & 3 & 4 & 5 & 6 & 7 \\
\hline Medios de comunicación & 1 & 2 & 3 & 4 & 5 & 6 & 7 \\
\hline Sindicatos & 1 & 2 & 3 & 4 & 5 & 6 & 7 \\
\hline Futuras generaciones & 1 & 2 & 3 & 4 & 5 & 6 & 7 \\
\hline Grupos feministas y/o asociaciones de mujeres & 1 & 2 & 3 & 4 & 5 & 6 & 7 \\
\hline
\end{tabular}


Características propias de la empresa

Marque por favor la casilla correspondiente para cada una de las siguientes cuestiones:

\begin{tabular}{|c|c|c|c|}
\hline Edad de la empresa en años & $\begin{array}{c}\text { Menos } \\
\text { de } 10\end{array}$ & $\begin{array}{c}\text { Entre } 10 \\
\text { y } 20\end{array}$ & $\begin{array}{l}\text { Más } \\
\text { de } 20\end{array}$ \\
\hline \multicolumn{2}{|l|}{ Código CNAE de su empresa (ver anexo) } & & \\
\hline \multicolumn{2}{|l|}{ Número total de trabajadores en plantilla } & & \\
\hline \multicolumn{2}{|l|}{ Porcentaje de mujeres en plantilla } & & \\
\hline \multicolumn{2}{|l|}{ Número de personas que componen el equipo de dirección } & & \\
\hline \multicolumn{2}{|l|}{ Porcentaje de mujeres en la dirección } & & \\
\hline \multicolumn{2}{|l|}{ Número de personas que componen el consejo de administración } & & \\
\hline \multicolumn{2}{|l|}{ Porcentaje de mujeres en el consejo } & & \\
\hline ¿Puede indicarnos su sexo? & Hombre & Mujer & \\
\hline ¿Ha elaborado su empresa un "plan de igualdad”? & $\mathrm{Si}$ & No & \\
\hline $\begin{array}{l}\text { ¿Han incluido algún informe sobre igualdad de oportunidades } \\
\text { entre mujeres y hombres en sus memorias anuales? }\end{array}$ & $\mathrm{Si}$ & No & \\
\hline
\end{tabular}

\section{MUCHAS GRACIAS POR SU COLABORACION}

Por favor, remita este cuestionario cumplimentado dentro del sobre adjunto a: XXXXXXXXXXXXXXXX Para cualquier consulta puede dejar un mensaje en la dirección de correo electrónico XXXXXXXX y nos pondremos en contacto con usted. También puede llamar si lo desea al teléfono XXXXXXX. 